\title{
MÉTODOS DE EXTRACCIÓN DE BORO Y RESPUESTA DEL JAÚL A SU ADICIÓN EN ANDISOLES DE COSTA RICA
}

\author{
Manuel Segura**, Álvaro Castillo**, Alfredo Alvarado ${ }^{1 / *}$ \\ Palabras clave: Métodos de extracción, boro, jaúl, andisoles, Alnus acuminata. \\ Keywords: Extraction methods, boron, alder, andisols, Alnus acuminata.
}

Recibido: 07/05/10

Aceptado: 29/09/10

\section{RESUMEN}

Se estudió el efecto de la adición de B en Andisoles sobre las variables altura, diámetro y volumen de los árboles en 5 plantaciones de Alnus acuminata de edad inicial en años de 0,5 en Hermanas Callejas (HC), 1 en Lechería Experimental (LE), 3 en Rosa Marta Cubero (RMC), 5 en Landelina (L) y 7 en Carter (C); a la última evaluación, las plantaciones tenían 3 años más de edad. El B se suplió como Boronato y se aplicó al voleo en cantidades de 0, 2, 4, 6 y 8 kg.ha- ${ }^{-1}$ para determinar su efecto residual a los 14 meses después de aplicado. El B adicionado incrementó su disponibilidad en el suelo, incremento que puede detectarse mediante extracción con agua caliente presurizada o con Mehlich 3. Los valores de correlación entre el B aplicado y el B extraído por el método de Mehlich 3 son mejores que los obtenidos con el método de agua caliente presurizada. La incorporación de 0 a 8 kg.ha-1 del elemento, no causó ningún incremento en las variables dasométricas del jaúl en los sitios comparados, excepto por un incremento significativo en volumen de madera en la plantación de Landelina (8 años de edad). La poca o ninguna respuesta encontrada, se atribuye a que el contenido inicial de $\mathrm{B}$ disponible en el suelo osciló entre 0,19-1,00 mg.kg-1, valores en su mayoría sobre el valor crítico de $0,5 \mathrm{mg} \cdot \mathrm{kg}^{-1}$ mencionado para cultivos en Andisoles y a un bajo requerimiento

1 Autor para correspondencia. Correo electrónico: alfredo.alvarado@@ucr.ac.cr

Centro de Investigaciones Agronómicas, Universidad de Costa Rica. San José, Costa Rica.

\section{ABSTRACT}

Soil boron extraction methods and alder response to boron additions in Andisols of Costa Rica. The effect of B to Andisols on tree height, diameter and volume of 5 Alnus acuminata plantations was studied. Plantation initial ages in years were: 0.5 at Hermanas Callejas (HC), 1 at Lechería Experimental (LE), 3 at Rosa Marta Cubero (RMC), 5 at Landelina (L) and 7 at Carter (C); at the end of the experiment plantations were 3 years older. B was supplied as Boronate and broadcasted at rates of $0,2,4,6$ and $8 \mathrm{~kg}$ ha ${ }^{-1}$; the residual effect of fertilizer was determined 14 months after its application. The fertilizer added incremented B availability in the soils, as measured with pressurized hot water and Mehlich 3 methods. $\mathrm{R}^{2}$ values obtained were higher with correlations obtained by the Mehlich 3 procedure. The B applied did not cause increments of dasometric variables measured, except for a significant increase in volume of wood at Landelina (8 year old plantation). The lack of response in growth variables to $\mathrm{B}$ addition are attributed to the initial $\mathrm{B}$ availability in the soils (0.19-1.00 mg. $\left.\mathrm{kg}^{-1}\right)$, most values above the critical value of $0.5 \mathrm{mg} \cdot \mathrm{kg}^{-1}$ mentioned for crops in Andisols, and the low requirement of $\mathrm{B}$ in alder. Foliar concentration of B showed a slight tendency to increase with growing

\footnotetext{
** Compañía Nacional de Fuerza y Luz, San José, Costa Rica.
} 
de B por parte del jaúl. La concentración foliar de B tendió a aumentar ligeramente conforme se elevó la cantidad de B adicionada al suelo, mientras que la concentración de $\mathrm{N}$ foliar no mostró ninguna tendencia.

\section{INTRODUCCIÓN}

La deficiencia de $\mathrm{B}$ en árboles maderables ocurre en muchos países, especialmente en las plantaciones con especies exóticas de eucalipto y pino, así como en sitios de plantaciones nativas en suelos alterados por aplicaciones de macronutrimentos, quemas y erosión (Stone 1990, Lehto et al. 2010). La deficiencia de B es común en suelos derivados de cenizas volcánicas (Andisoles) de composición basáltica con $\mathrm{pH}<5,0$ (Shoji et al. 1993), en particular cuando estos son arenosos y se encuentran en regiones con precipitación pluvial elevada (Goldberg y Su 2007). Al resumir la información de B disponible en Andisoles de Japón y Hawaii, Shoji et al. (1993) consideran como niveles bajos de $\mathrm{B}$ extraídos con agua caliente, concentraciones en el ámbito 0,2-0,5 mg. $\mathrm{kg}^{-1}$ y como nivel crítico para cultivos el valor de $1 \mathrm{mg} \cdot \mathrm{kg}^{-1}$; en Andisoles de Ecuador, Tollenaar (1966) considera como nivel crítico el valor de 0,20 mg. $\mathrm{kg}^{-1}$ para el cultivo de cacao. Bornemisza (1982) menciona que la adsorción de B en Andisoles no es reversible y por tanto los suelos de este origen suelen ser deficientes en B, sobre todo si son sobreencalados.

La deficiencia de B en Andisoles de América Latina, es común en plantaciones de ciprés, pino y eucaliptos en Colombia (Ladrach 1980a,b, Cannon 1981a,b) y de pino en Chile (Gonzales et al. 1983); la deficiencia se corrige con aplicaciones de 1-8 kg.ha-1 de $\mathrm{B}$ el valor más alto para suelos de textura fina, con un efecto residual de 4 años. En Andisoles de Chile, la adición de 13-20 g BNC (boronitrocalcita).árbol ${ }^{-1}$ produce $^{2}$ amounts of B applied to the soil, while $\mathrm{N}$ foliar concentration did not show any tendency after $\mathrm{B}$ addition to the soil.

un incremento de biomasa del $10 \%$ en $P$. radiata (Gonzales et al. 1983).

En cultivos tropicales, la deficiencia de B en Andisoles de la costa de Ecuador, se atribuye a la combinación de niveles bajos de $\mathrm{B}$ en el suelo $\left(0,18-0,23 \mathrm{mg}\right.$ B soluble en agua. $\mathrm{kg}^{-1}$ de suelo), altos contenidos de $\mathrm{Ca}$ en el suelo y en períodos prolongados de sequía (Tollenaar 1966); la deficiencia logró corregirse para el cultivo de cacao con la adición de 250 g.arbol ${ }^{-1}$ de "Fritted Trace Elements" con un 10\% de B. En Andisoles de Costa Rica, Rímolo (1970) encontró que el B disponible disminuye al aumentar el $\mathrm{pH}\left(\mathrm{CaCl}_{2}\right)$ de 4,2 a 6,0, debido a que la capacidad máxima de adsorción del elemento aumenta en el mismo rango de $\mathrm{pH}$. La respuesta a la adición de $\mathrm{B}$ en el país se ha documentado para café (Pérez et al. 1957, Pérez 1957, Ramírez 1998), brócoli (Hernández 1977), coliflor (Araya 1978, Jiménez y Cordero 1988) y caña de azúcar (Chaves y Alvarado 1994).

En plantaciones de jaúl (Alnus acuminata) en Andisoles de Costa Rica, Segura et al. (2005) encontraron que el B acumulado en la biomasa aérea en árboles de 2, 4 y 7 años es de 33, 90 y 337 g.ha ${ }^{-1}$, con un incremento medio anual de B de 16, 29 y 82 g.ha $^{-1}$. año $^{-1}$; lo anterior demuestra que, con la edad, el jaúl requiere una mayor cantidad de B para llenar sus necesidades fisiológicas, que acumuló el 49\% del B absorbido en las hojas a la edad de 2 años y el $72 \%$ del mismo en los tallos a la edad de 7 años. En las mismas plantaciones, Segura et al. (2006) mencionan como ligeramente baja, adecuada y ligeramente alta, valores de B foliar de 5-11, 12-21 y 22-28 mg.kg ${ }^{-1}$, respectivamente. No se conoce información referente a la 
respuesta de A. acuminata a la adición de $\mathrm{B}$ en ningún tipo de suelo.

El objetivo del presente trabajo es estudiar la respuesta del jaúl a la adición de dosis crecientes de $\mathrm{B}$ al suelo en plantaciones de $0,5,1,0$, 3,0, 6,0 y 8,0 años de edad inicial (serie falsa de tiempo) en Andisoles de la Cordillera Central de Costa Rica.

\section{MATERIALES Y MÉTODOS}

\section{Variables ambientales}

La zona de estudio se ubica entre 1542 y 2062 msnm, en la zona de vida Bosque Muy Húmedo Montano Bajo (bmh-MB). La precipitación promedio anual es de $2500 \mathrm{~mm}$ y la temperatura media ambiente oscila durante el año entre 12,0 y $22,5^{\circ} \mathrm{C}$; la humedad relativa de la zona es alta y la neblina es frecuente, con vientos leves (Montoya 1999). El estudio se realizó en 5 plantaciones de A. acuminata de edad inicial en años de 0,5 en Hermanas Callejas (HC), 1 en Lechería Experimental (LE), 3 en Rosa Marta Cubero (RMC), 5 en Landelina (L) y 7 en Carter (C). Al trabajar con plantaciones de diferente edad, se pretende establecer una "serie falsa de tiempo", para estimar en un tiempo corto, el efecto de la adición de $\mathrm{B}$ en función de los requerimientos del elemento en árboles de edad creciente.
La plantaciones bajo estudios se consideran como de Clase I y II para el crecimiento de la especie según los criterios desarrollados por Camacho y Murillo (1986), en Andisoles de los cantones Vásquez de Coronado y Goicoechea. Las mismas se encuentran en relieves moderadamente y fuertemente ondulados con la pendiente de los terrenos donde se ubicaron los ensayos de $30-40 \%$ en HC, de $60 \%$ en LE, de $40 \%$ en RMC y L y de $10-20 \%$ en C. Todas las plantaciones, se establecieron a una distancia de $3,5 \times 3,5 \mathrm{~m}$, para una densidad de 816 árboles.ha $^{-1}$, excepto HC en la cual los árboles fueron establecidos a una distancia de 3,5x3,0 m, para una densidad de 952 árboles.ha ${ }^{-1}$. Los sitios están bajo el cuidado del Departamento de Recursos Naturales, Dirección Ambiental de la Compañía Nacional de Fuerza y Luz (CNFL).

Los suelos de las fincas bajo estudio clasifican como Typic Hapludands (Fincas LE, RMC, HC y C) y Thaptic Epiaquands (Finca L), los cuales se caracterizan por tener altos contenidos de arena (63-68\%) (Cuadro 1). En general los suelos son de fertilidad baja (CICE $\left.<6,6 \mathrm{cmol}(+) . \mathrm{l}^{-1}\right)$, sin problemas de acidez, pero con niveles bajos (Fincas HC y RMC) a medios (Fincas L, LE y C) de $\mathrm{P}$ disponible. A pesar del bajo grado de meteorización de las cenizas volcánicas (3-9\% arcilla), el contenido de materia orgánica de los suelos es moderadamente bajo $(3,2-4,8 \%)$ a medio $(5,7-6,4 \%)$.

Cuadro 1. Principales características de los suelos de las fincas bajo estudio.

\begin{tabular}{|c|c|c|c|c|c|c|c|c|c|c|c|c|c|c|c|c|}
\hline Finca & $\begin{array}{c}\mathrm{pH} \\
\mathrm{H}_{2} \mathrm{O}\end{array}$ & \multicolumn{5}{|c|}{$\operatorname{cmol}(+) .1^{-1}$} & \multicolumn{6}{|c|}{ mg..$^{-1}$} & M.O. & Arena & Limo & Arcilla \\
\hline L (N 5) & 5,4 & 2,28 & 0,80 & 0,13 & 0,41 & 3,62 & 11 & 10 & 13 & 222 & 3 & 4 & 6,23 & 69 & 28 & 3 \\
\hline $\mathrm{HC}(\mathrm{N} 4)$ & 5,7 & 2,83 & 1,03 & 0,25 & 0,33 & 4,43 & 4 & 12 & 16 & 128 & 3 & 4 & 5,68 & 63 & 30 & 7 \\
\hline LE (N 5) & 6,2 & 2,58 & 1,09 & 0,27 & 0,30 & 4,23 & 11 & 12 & 8 & 122 & 2 & 3 & 3,20 & 67 & 27 & 5 \\
\hline C (N 5) & 5,6 & 4,07 & 1,15 & 0,41 & 030 & 5,93 & 14 & 12 & 13 & 193 & 4 & 4 & 6,35 & 63 & 30 & 6 \\
\hline RMC (N 5) & 5,8 & 3,92 & 1,33 & 0,73 & 0,63 & 6,61 & 3 & 11 & 16 & 148 & 2 & 3 & 4,80 & 63 & 28 & 9 \\
\hline
\end{tabular}




\section{Recolección y preparación de muestras}

Se recolectaron muestras compuestas de suelo al inicio del experimento y posteriormente en 1 y 8 meses, se realizó el último muestreo en cada una de las 3 plantaciones. Las muestras se tomaron con barreno y el método de muestreo propuesto por laboratorio de suelos del CIA (Henríquez et al. 1995), el cual consiste en realizar un muestreo en zig-zag en 6 sitios por tratamiento (submuestras) a una profundidad de 0 a $20 \mathrm{~cm} \mathrm{y}$ en la zona donde se encuentran la mayoría de las raíces de los árboles. Las submuestras representaban las 3 repeticiones de cada tratamiento en cada localidad y se guardaron en bolsas plásticas para obtener un total de $0,5 \mathrm{~kg}$ de suelo.

Las muestras recolectadas se enviaron al Laboratorio de Suelos del Centro de Investigaciones Agronómicas de la Universidad de Costa Rica, donde fueron analizadas por $\mathrm{pH}$ en agua, $\mathrm{Ca}, \mathrm{Mg}, \mathrm{K}$, acidez intercambiable, $\mathrm{P}, \mathrm{Fe}, \mathrm{Cu}, \mathrm{Zn}$, $\mathrm{Mn}, \mathrm{Al}$ intercambiable y $\mathrm{N}$ total con el uso de la metodología de Díaz-Romeu y Hunter (1978). La materia orgánica se determinó por el método de combustión húmeda de Walkey y Black, descrito por Briceño y Pacheco (1984). Para determinar el tipo de textura se utilizó el método de Bouyoucos modificado tal y como viene descrito en la metodología escrita por Forsythe (1975), con el empleo de la hoja de cálculo Anapart desarrollada por el Dr. W. Forsythe para obtener los porcentajes de partículas de diferente tamaño.

El B disponible en el suelo se determinó mediante extracción con agua caliente presurizada, según el método de Berger y Truog (1939) modificado por Masís F. 2009 (Comunicación personal) que consiste en pesar una muestra de 2,0 g de suelo (cribado en malla $2 \mathrm{~mm}$ ), colocarlo en un envase de microondas con $10 \mathrm{ml}$ agua, programar el microondas para elevar la temperatura cada 5 min hasta alcanzar los $100^{\circ} \mathrm{C}$, mantener la temperatura constante a $100^{\circ} \mathrm{C}$ por $5 \mathrm{~min}$ y aplicar enfriamiento cada 5 min hasta alcanzar la temperatura ambiente. Al finalizar la extracción con agua caliente, filtrar con papel de filtro Advantec $\mathrm{N}^{\mathrm{o}}$. 5A el contenido de los envases. A modo de comparación, el $\mathrm{B}$ disponible también se extrajo con el empleo del método de Mehlich 3 (Mehlich 1984) a partir de una muestra de 2,5 $\mathrm{g}$ de suelo, agitada con $25 \mathrm{ml}$ de solución (HOAc $0,2 \mathrm{~N} ; \mathrm{NH}_{4} \mathrm{NO}_{3} 0,25 \mathrm{~N} ; \mathrm{NH}_{4} \mathrm{~F} 0,015 \mathrm{~N} ; \mathrm{HNO}_{3}$ $0,013 \mathrm{~N}$; EDTA $0,001 \mathrm{M}$; pH 2,5) por $5 \mathrm{~min}$ y con papel de filtro Advantec No. $5 \mathrm{~A}$ el contenido de los envases. En ambos casos, el B se determina a partir del filtrado, en un espectrofotómetro de emisión atómica (ICP) con plasma con una longitud de onda igual a $249,772 \mathrm{~nm}$, y una curva con concentraciones de $0,0,25,0,50,1,0$ y $2,0{\mathrm{mg} . \mathrm{I}^{-1}}^{-1}$ de $\mathrm{B}$ en el caso de la extracción con agua caliente y de $0,0,1,0,2$ y $0,4 \mathrm{mg} . \mathrm{l}^{-1}$ de B para la extracción con Mehlich 3.

Para la determinación del contenido foliar de los elementos, se tomó tejido de todos los tratamientos en las diferentes fincas y además se combinaron las muestras de las 3 repeticiones por finca, debido a que no hubo respuesta a las variables de crecimiento ni efecto de la adición de B sobre el B extraíble del suelo por ningún método. Las muestras recolectadas se enviaron al Laboratorio de Suelos del Centro de Investigaciones Agronómicas de la Universidad de Costa Rica, donde fueron analizadas. Las muestras se transportaron al Centro de Investigaciones Agronómicas de la Universidad de Costa Rica y se analizaron para $\mathrm{N}$ total, $\mathrm{P}, \mathrm{Ca}, \mathrm{Mg}, \mathrm{K}, \mathrm{S}, \mathrm{Fe}$, $\mathrm{Cu}, \mathrm{Zn}, \mathrm{Mn}$, y B por combustión húmeda con la utilización de la metodología descrita por Operation Manual, Microwave Accelerated Reaction System (2001) y Manual Digestion Application Notes (2001).

Como variables dasométricas, se midieron el diámetro a la altura del pecho $(1,3 \mathrm{~m})$ y la altura de los árboles por el método indirecto descrito por Jumkov (1994). También se determinó el área basal $\left(\mathrm{m}^{2} \cdot \mathrm{ha}^{-1}\right)$, el rendimiento $\left(\mathrm{m}^{3} \cdot \mathrm{ha}^{-1}\right)$ y el volumen de los árboles, con la fórmula de Schumacher modificada para las parcelas permanentes de jaúl en la cuenca alta del río Virilla (Castillo A. 2005. Comunicación personal).

Las mediciones se iniciaron 14 meses después de la aplicación de B y con los valores obtenidos se calculó el Incremento Promedio Relativo (IPR) como el valor promedio de las diferencias 
entre los valores de las variables de crecimiento bajo cada tratamiento con $\mathrm{B}$, menos el valor de la misma variable en la parcela testigo: $\mathrm{IPR}=((\mathrm{B} 2-$ $\mathrm{B} 0)+(\mathrm{B} 4-\mathrm{B} 0)+(\mathrm{B} 6-\mathrm{B} 0)+\mathrm{B} 8-\mathrm{B} 0)) / 4$, esto en cada una de las fincas (5 edades). De esta manera, se puede estimar el efecto de la adición de $\mathrm{B}$ a cada edad (SITIO).

\section{Aplicación de B al suelo}

En las 3 plantaciones se aplicaron dosis crecientes de $\mathrm{B}$ con el empleo de Boronato (0-00-0-9,92), borato de sodio y calcio que permite suplir B a corto y mediano plazo en forma inmediata y continúa. El fertilizante se aplicó en cantidades de 0, 2, 4, 6 y 8 kg.ha- ${ }^{-1}$ de B con un diseño de bloques completos al azar. Para facilitar la aplicación del producto al voleo, este se mezcló con suelo recolectado en cada una de las plantaciones en una relación de $12 \mathrm{~kg}$ de suelo en las parcelas pequeñas y $24 \mathrm{~kg}$ en las parcelas grandes. Las parcelas grandes corresponden a las de las plantaciones Rosa Marta Cubero y César Cárter, sembradas a 3,5×3,5 m, en las cuales se aumentó el tamaño de parcela útil para contar con al menos 10 árboles útiles, dada la muerte de algunos árboles en las plantaciones originales; las parcelas pequeñas (Lechería Experimental y Landelina) estaban constituidas por un tamaño total de 36 árboles $(6 \times 6)$ en un área de $441 \mathrm{~m}^{2}$ y la parcela útil constó de 16 árboles $(4 x 4)$ y 196 m²$^{2}$. En la plantación Hermanas Calleja el espaciamiento entre árboles fue de $3 \times 3$ total de 36 árboles $(6 \times 6)$ en un área de $324 \mathrm{~m}^{2}$ y la parcela útil constó de 16 árboles $(4 \mathrm{x} 4)$ y $144 \mathrm{~m}^{2}$. Los bloques fueron ubicados en las áreas con condiciones edáficas similares y árboles que representaban la altura y diámetro de los árboles dominantes y codominantes, de modo que la respuesta al tratamiento fuera lo más representativa posible.

\section{Diseño experimental y análisis estadístico}

El diseño experimental consistió en la distribución de 5 tratamientos de $\mathrm{B}$, en 3 repeticiones (bloques) en cada una de las 5 fincas (edades), para un total de 15 parcelas por plantación. Los bloques se ubicaron en áreas con condiciones edáficas similares y árboles que representaban la altura y diámetro de los árboles dominantes y co-dominantes, de modo que la respuesta al tratamiento fuera lo más representativa posible.

Se realizó un análisis gráfico de dispersión preliminar para observar las tendencias entre las variables comparadas y otro de varianza para las variables diámetro, altura, volumen y su interacción, todo en función de los tratamientos de B aplicados. Además se realizó un análisis de regresión lineal entre el diámetro vs altura y el diámetro vs volumen. El análisis de los datos se realizó solamente a partir de los arboles dominantes y co-dominantes, es decir, se eliminaron los árboles suprimidos.

\section{RESULTADOS Y DISCUSIÓN}

\section{Efecto del B aplicado al suelo sobre la cantidad de $B$ extraíble}

Para estimar el B disponible en el suelo, se determinó su contenido con el empleo el método de agua caliente presurizado y reconocido por varios autores como el mejor (Shoji et al. 1993, Goldberg y Su 2007) y otro método de extracción de uso corriente en los laboratorios del Centro de Investigaciones Agronómicas de la Universidad de Costa Rica (Mehlich 3). En el Cuadro 2 se observa la cantidad de B extraída por ambos métodos en los 5 suelos comparados, en muestras tomadas 20 meses después de la aplicación del elemento al voleo.

Al comparar los datos de B extraído en todos los sitios por ambos métodos, se observa que los contenidos de B en agua caliente son en promedio más elevados que los extraídos con Mehlich $3\left(0,60 \mathrm{mg} \cdot \mathrm{kg}^{-1}\right.$ vs $\left.0,37 \mathrm{mg} \cdot \mathrm{l}^{-1}\right)$ y que la correlación entre los 2 métodos de extracción es baja (Figura 1).

La cantidad de B extraído con los 2 métodos comparados, en función del B aplicado por sitio (finca), muestra que la relación entre lo aplicado y lo extraído es mayormente cuadrática, 
Cuadro 2. Cantidad de B extraíble en agua caliente y Mehlich 3 en función del B aplicado como fertilizante al suelo en las 5 fincas comparadas.

\begin{tabular}{|c|c|c|c|c|c|c|}
\hline \multirow{2}{*}{ Finca } & \multicolumn{6}{|c|}{ Cantidad de B $\left(\mathrm{kg} \cdot \mathrm{ha}^{-1}\right)$ aplicado al suelo } \\
\hline & 0,00 & 2,00 & 4,00 & 6,00 & 8,00 & Gran Prom. \\
\hline \multicolumn{7}{|c|}{ B extraíble Mehlich 3 (mg.1-1) } \\
\hline $\mathrm{HC}$ & $0,18( \pm 0,04)$ & $0,26( \pm 0,01)$ & $0,32( \pm 0,07)$ & $0,30( \pm 0,05)$ & $0,32( \pm 0,05)$ & 0,28 \\
\hline LE & $0,21( \pm 0,07)$ & $0,22( \pm 0,01)$ & $0,28( \pm 0,04)$ & $0,41( \pm 0,08)$ & $0,33( \pm 0,08)$ & 0,29 \\
\hline $\mathrm{L}$ & $0,23( \pm 0,06)$ & $0,28( \pm 0,06)$ & $0,29( \pm 0,01)$ & $0,33( \pm 0,12)$ & $0,30( \pm 0,04)$ & 0,29 \\
\hline $\mathrm{C}$ & $0,27( \pm 0,02)$ & $0,31( \pm 0,09)$ & $0,39( \pm 0,12)$ & $0,38( \pm 0,07)$ & $0,33( \pm 0,04)$ & 0,34 \\
\hline $\mathrm{RMC}$ & $0,28( \pm 0,03)$ & $0,40( \pm 0,10)$ & $0,37( \pm 0,04)$ & $0,56( \pm 0,01)$ & $0,50( \pm 0,12)$ & 0,42 \\
\hline Promedio & 0,23 & 0,30 & 0,33 & 0,40 & 0,36 & 0,32 \\
\hline \multicolumn{7}{|c|}{ B extraíble agua caliente $\left(\mathrm{mg} . \mathrm{l}^{-1}\right)$} \\
\hline $\mathrm{HC}$ & $0,32( \pm 0,07)$ & $0,43( \pm 0,05)$ & $0,60( \pm 0,14)$ & $0,47( \pm 0,14)$ & $0,61( \pm 0,09)$ & 0,48 \\
\hline LE & $0,23( \pm 0,06)$ & $0,37( \pm 0,10)$ & $0,45( \pm 0,15)$ & $0,55( \pm 0,23)$ & $0,40( \pm 0,16)$ & 0,40 \\
\hline $\mathrm{L}$ & $0,43( \pm 0,09)$ & $0,49( \pm 0,11)$ & $0,49( \pm 0,06)$ & $0,47( \pm 0,04)$ & $0,54( \pm 0,12)$ & 0,48 \\
\hline $\mathrm{C}$ & $0,55( \pm 0,03)$ & $0,63( \pm 0,08)$ & $0,78( \pm 0,10)$ & $0,76( \pm 0,15)$ & $0,72( \pm 0,02)$ & 0,69 \\
\hline $\mathrm{RMC}$ & $0,73( \pm 0,24)$ & $1,18( \pm 0,45)$ & $1,11( \pm 0,46)$ & $0,71( \pm 0,15)$ & $1,01( \pm 0,17)$ & 0,95 \\
\hline Promedio & 0,45 & 0,62 & 0,69 & 0,59 & 0,66 & 0,60 \\
\hline
\end{tabular}

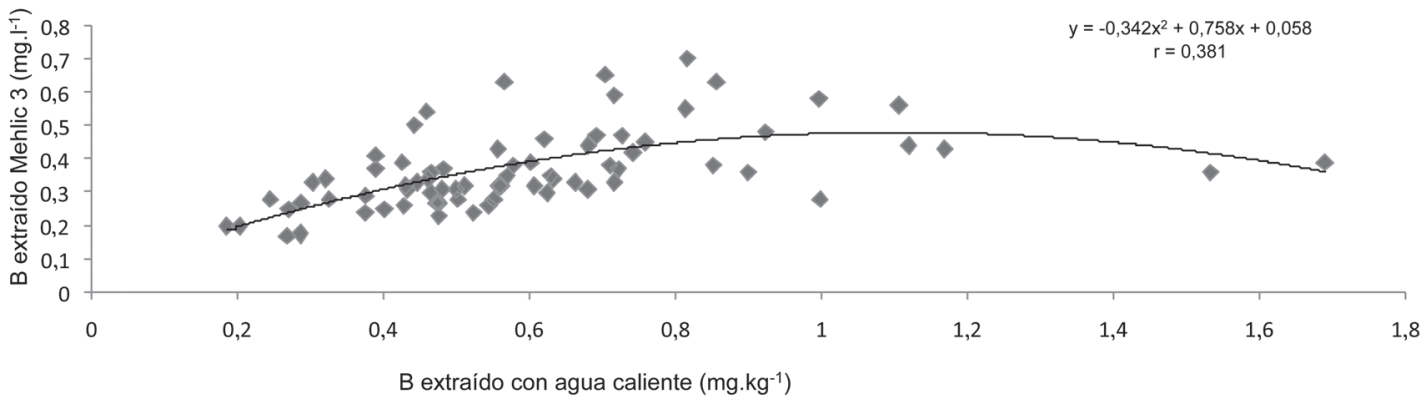

Fig. 1. Correlación entre el contenido de B extraído con agua caliente y con Mehlich 3 en los suelos bajo estudio.

aunque el coeficiente de regresión $\left(\mathrm{R}^{2}\right)$ puede ser alto o bajo, según sea el método o el suelo de la finca bajo análisis. Al extraer el B disponible con agua caliente presurizada, se encontró valores de $\mathrm{R}^{2}$ altos en los suelos de las finca LE y $\mathrm{C}$, medios en HC y bajos LE y RMC, mientras que al realizar la extracción con Mehlich 3, los valores de $\mathrm{R}^{2}$ fueron altos en las fincas $\mathrm{HC}$, LE y $\mathrm{C}$ y medios en RMC y LE (Cuadro 3). Por lo anterior, se sugiere que el método de Mehlich 3 estima de mejor manera el contenido de $\mathrm{B}$ disponible en los Andisoles bajo estudio. 
Cuadro 3. Modelos de ajuste y coeficientes de correlación entre el B aplicado al suelo y el extraído con agua caliente presurizada y Mehlich 3 en 5 suelos a los que se les aplicó 5 niveles de B al voleo (20 meses después de la aplicación).

\begin{tabular}{llc|cc}
\hline & \multicolumn{3}{c}{ Mejor modelo de ajuste } & \\
Finca & \multicolumn{2}{c}{ Agua caliente presurizado } & & \multicolumn{2}{c}{ Mehlich 3} \\
& \multicolumn{2}{c}{ Ecuación } & $\mathrm{R}^{2}$ & \multicolumn{2}{c}{ Ecuación } & $\mathrm{R}^{2}$ \\
\hline HC & $\mathrm{y}=-0,004 \mathrm{x}^{2}+0,065 \mathrm{x}+0,326$ & 0,709 & $\mathrm{y}=-0,003 \mathrm{x}^{2}+0,049 \mathrm{x}+0,207$ & 0,913 \\
LE & $\mathrm{y}=-0,010 \mathrm{x}^{2}+0,107 \mathrm{x}+0,218$ & 0,905 & $\mathrm{y}=-0,002 \mathrm{x}^{2}+0,040 \mathrm{x}+0,218$ & 0,661 \\
RMC & $\mathrm{y}=-0,011 \mathrm{x}^{2}+0,094 \mathrm{x}+0,837$ & 0,152 & $\mathrm{y}=-0,002 \mathrm{x}^{2}+0,056 \mathrm{x}+0,318$ & 0,726 \\
LE & $\mathrm{y}=0,000008 \mathrm{x}^{2}+0,010 \mathrm{x}+0,442$ & 0,576 & $\mathrm{y}=-0,002 \mathrm{x}^{2}+0,032 \mathrm{x}+0,258$ & 0,906 \\
C & $\mathrm{y}=-0,007 \mathrm{x}^{2}+0,081 \mathrm{x}+0,538330$ & 0,926 & $\mathrm{y}=-0,005 \mathrm{x}^{2}+0,055 \mathrm{x}+0,289$ & 0,919 \\
\hline
\end{tabular}

B extraíble con agua caliente: En la Figura 2 se observa la relación entre el B aplicado al suelo y el extraído con agua caliente en cada finca; se considera el valor promedio de 3 repeticiones para cada nivel de $\mathrm{B}$ adicionado, de manera que se reduce al mínimo el efecto de sitio y se resalta el efecto de los niveles de $\mathrm{B}$ aplicados, 20 meses después de su aplicación al voleo. El B disponible en el suelo por este método cuando no se aplicó el elemento como fertilizante, osciló entre $0,19-1,00 \mathrm{mg} \cdot \mathrm{kg}^{-1}$ en las 5 fincas comparadas; valores considerados como bajos (LE, HC y L), marginal (C) y adecuado (RMC), según los criterios de Shoji et al. (1993). La adición de B al suelo, elevó ligeramente y de manera no significativa los niveles de $\mathrm{B}$ extraíble en todas las fincas hasta el nivel de $6 \mathrm{~kg} \cdot \mathrm{ha}^{-1}$ de $\mathrm{B}$. La adición de $6 \mathrm{~kg} \cdot \mathrm{ha}^{-1}$ de $\mathrm{B}$ fue suficiente para elevar el contenido de $\mathrm{B}$ en el suelo a niveles superiores a $0,5 \mathrm{mg} \cdot \mathrm{kg}^{-1}$ de $\mathrm{B}$ en la mayoría de los sitios, con un efecto de mayor magnitud en las fincas $\mathrm{C}$ y $\mathrm{RMC}$, en las cuales el promedio de B disponible fue mucho mayor que el encontrado en las fincas con menor CICE (L, HC y LE). El B disponible en el suelo aumentó de manera cuadrática en todos los casos, en unos con mayor grado de significancia estadística (C, LE y HC), aunque pudo ajustarse las relaciones a un modelo lineal si no

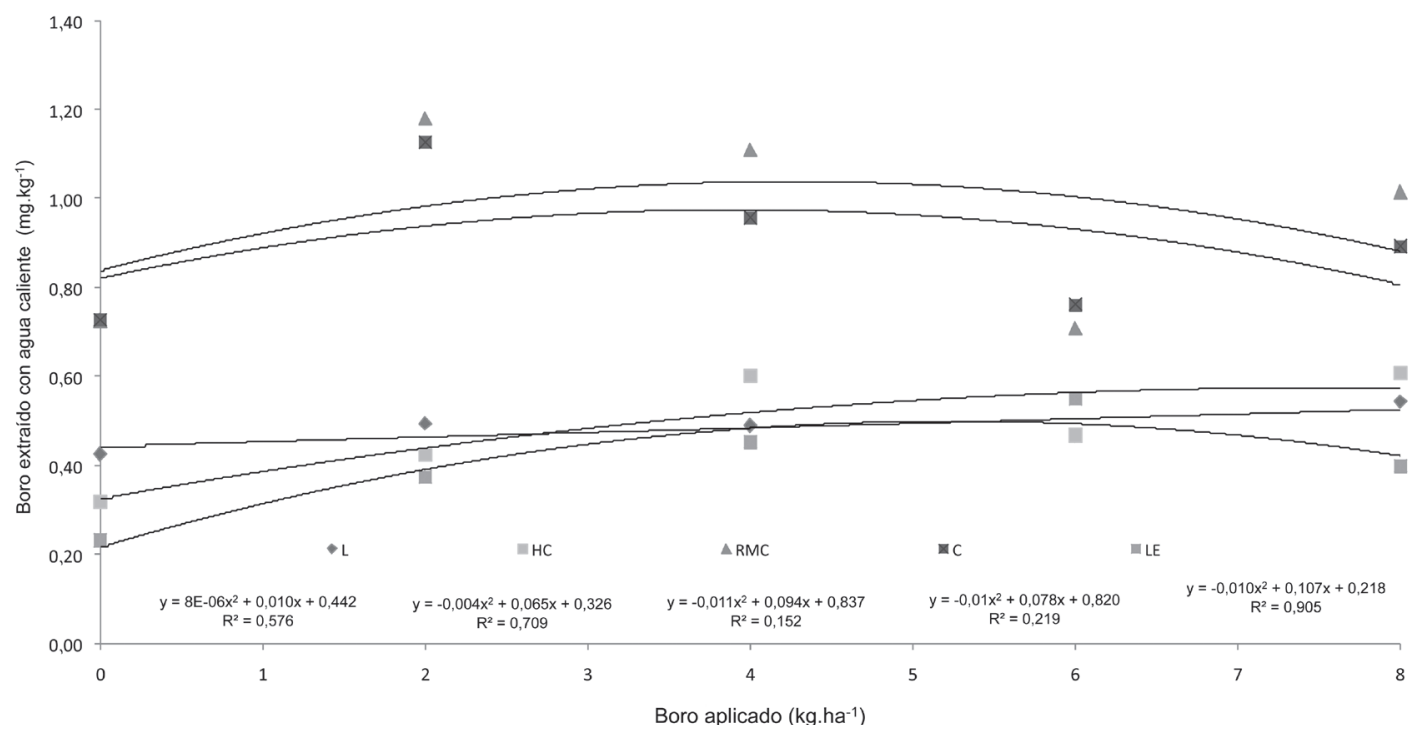

Fig. 2. Cantidad de B extraíble del suelo con agua caliente en función del B aplicado al suelo en 5 plantaciones de jaúl en Andisoles de Costa Rica. 
se hubiese considerado la adición de $8 \mathrm{~kg} \cdot \mathrm{ha}^{-1}$ de B; sin embargo, esta opción no se consideró pues equivaldría a ignorar datos de investigación de campo reales y confiables.

B extraíble con Mehlich 3: En la Figura 3 se observa la relación entre el B aplicado al suelo y el extraído con Mehlich 3. El B disponible por este método en el suelo cuando no se aplicó el elemento como fertilizante, osciló entre 0,18-0,28 mg..$^{-1}$ en las 5 fincas comparadas. La adición de
$\mathrm{B}$, elevó ligeramente y de manera no significativa los niveles de $\mathrm{B}$ extraíble en todas las fincas hasta el nivel de 6 kg.ha ${ }^{-1}$ de B este nivel fue suficiente para elevar el contenido de $\mathrm{B}$ en el suelo a niveles superiores a $0,3 \mathrm{mg} . \mathrm{l}^{-1}$ de B en la mayoría de los sitios, con un efecto de mayor magnitud en las fincas C y RMC. De forma individual, el B disponible en el suelo aumentó de manera cuadrática en todos los casos, en unos con mayor grado de significancia estadística (HC, L y C).

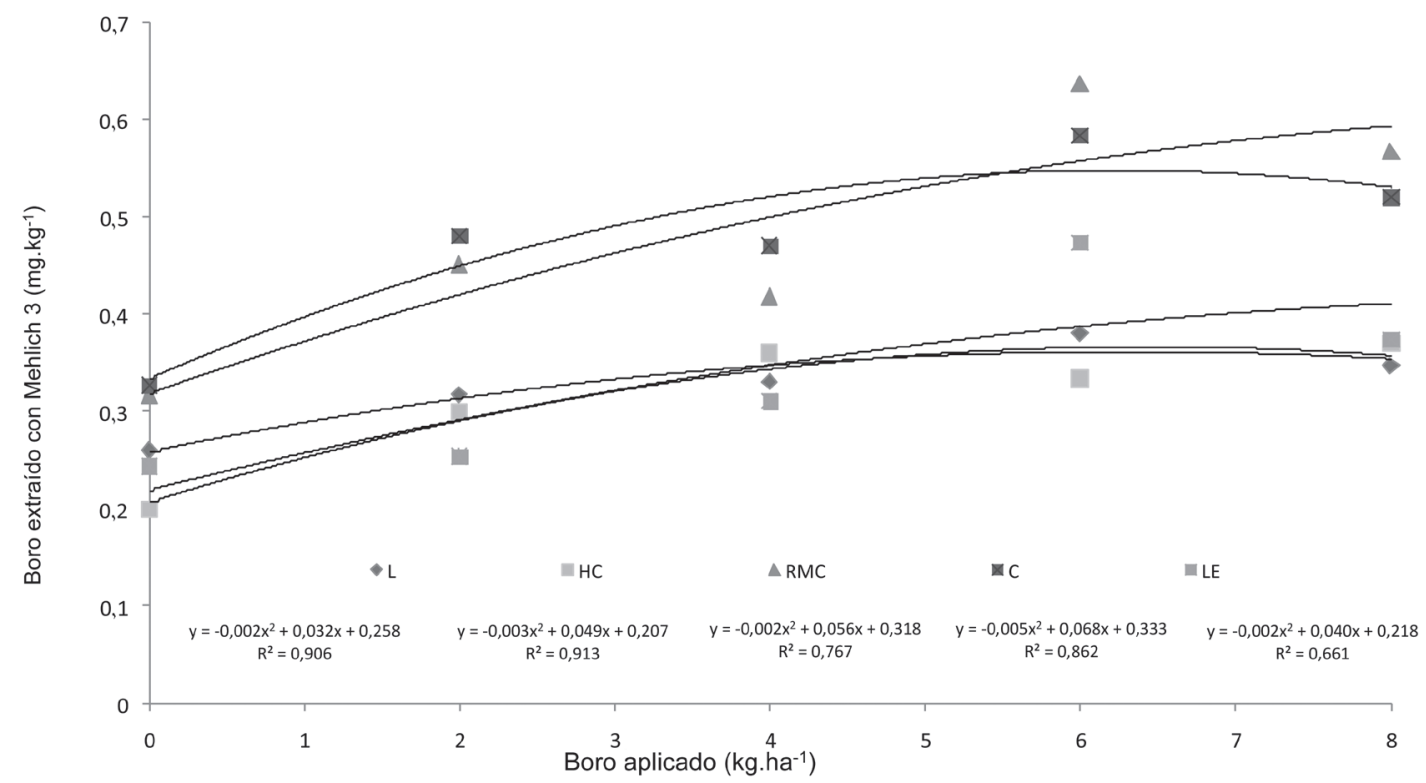

Fig.3. Cantidad de B extraíble del suelo con Mehlich 3 en función del B aplicado al suelo en 5 plantaciones de jaúl en Andisoles Costa Rica.

\section{Efecto de la adición de B sobre el diámetro, la} altura y el volumen de los árboles

Se observó una tendencia de aumento en la altura promedio por árbol con la aplicación de B en las plantaciones LE y RMC. Solamente en la finca L se encontró diferencias significativas en volumen como resultado de la aplicación de $\mathrm{B}$, donde se observó una tendencia de aumento en volumen hasta la dosis de $6 \mathrm{~kg} \cdot \mathrm{ha}^{-1}$ de B $(\mathrm{p}=0,0121)$ (Cuadro 4). La poca respuesta a la adición de $\mathrm{B}$ puede deberse a varias causas, entre ellas: 1) a que en algunos sitios el contenido de B disponible en el suelo era superior al nivel crítico mencionado en suelos similares de otros países (Shoji et al. 1993), 2) al hecho de que el B adicionado al suelo se lavara durante la estación lluviosa, dadas la predominancia de texturas arenosa de los suelos (Cuadro 1), como fuera reportado en condiciones similares en otros sitios (Goldberg y Su 2007), o 3) a que los requerimientos de B por parte del jaúl sean muy bajos. 
Cuadro 4. Efecto de la adición de B al suelo sobre las variables de crecimiento (después de 20 meses de aplicado) sobre las variables de crecimiento de jaúl de diferente edad en Andisoles de Costa Rica.

\begin{tabular}{|c|c|c|c|c|c|c|c|c|c|}
\hline \multirow[t]{2}{*}{ Sitio } & \multirow{2}{*}{$\begin{array}{c}\text { Edad inicial } \\
\text { (años) }\end{array}$} & \multicolumn{5}{|c|}{ Boro aplicado (kg.ha $\left.{ }^{-1}\right)$} & \multirow{2}{*}{$\begin{array}{c}\text { Incr. Rel.* } \\
\text { Test. (Prom.) }\end{array}$} & \multirow[t]{2}{*}{$\mathrm{pr}>\mathrm{F}$} & \multirow{2}{*}{$\begin{array}{c}\text { Desv. } \\
\text { Est. }\end{array}$} \\
\hline & & 0 & 2 & 4 & 6 & 8 & & & \\
\hline & & \multicolumn{8}{|c|}{ Altura de los árboles (m) } \\
\hline $\mathrm{HC}$ & 0,5 & 4,8 & 4,82 & 4,74 & 4,88 & 4,77 & 0 & 0,9995 & 0,05 \\
\hline LE & 1 & 10,79 & 11,64 & 11,05 & 10,94 & 10,27 & 0,19 & 0,2447 & 0,49 \\
\hline $\mathrm{RMC}$ & 3 & 11,86 & 11,5 & 12,38 & 12,78 & 12,76 & 0,49 & 0,1347 & 0,56 \\
\hline $\mathrm{L}$ & 5 & 19,36 & 20,2 & 20,39 & 20,17 & 20,79 & 1,02 & 0,194 & 0,52 \\
\hline \multirow[t]{2}{*}{$\mathrm{C}$} & 7 & 20,15 & 19,54 & 20,18 & 20,68 & 20,17 & $-0,01$ & 0,4408 & 0,41 \\
\hline & & \multicolumn{8}{|c|}{ Diámetro de los árboles $(\mathrm{cm})$} \\
\hline $\mathrm{HC}$ & 0,5 & 3,96 & 4,16 & 4,03 & 4,12 & 3,8 & 0,07 & 0,9908 & 0,14 \\
\hline LE & 1 & 11,08 & 11,13 & 11,7 & 11,38 & 10,56 & 0,11 & 0,6659 & 0,42 \\
\hline RMC & 3 & 11,21 & 11,22 & 11,22 & 11,2 & 11,14 & $-0,01$ & 1 & 0,03 \\
\hline $\mathrm{L}$ & 5 & 20,17 & 20,73 & 21,48 & 21,88 & 21,35 & 1,19 & 0,2074 & 0,67 \\
\hline \multirow[t]{2}{*}{$\mathrm{C}$} & 7 & 23,5 & 23,47 & 23,04 & 23,26 & 23,2 & $-0,25$ & 0,9729 & 0,19 \\
\hline & & \multicolumn{8}{|c|}{ Volumen de madera $\left(\mathrm{m}^{3}\right)$} \\
\hline $\mathrm{HC}$ & 0,5 & 0,0035 & 0,0038 & 0,0036 & 0,0041 & 0,0031 & 0,0002 & 1 & 0,0004 \\
\hline LE & 1 & 0,054 & 0,058 & 0,0634 & 0,0587 & 0,0468 & 0,0027 & 0,9607 & 0,0062 \\
\hline RMC & 3 & 0,0613 & 0,0598 & 0,0648 & 0,0665 & 0,0661 & 0,003 & 0,9976 & 0,003 \\
\hline $\mathrm{L}$ & 5 & 0,3111 & 0,342 & 0,3707 & 0,3857 & 0,3763 & 0,0576 & 0,0121 & 0,0305 \\
\hline $\mathrm{C}$ & 7 & 0,4455 & 0,4264 & 0,419 & 0,4448 & 0,4303 & $-0,0154$ & 0,7102 & 0,0116 \\
\hline
\end{tabular}

Des. Est=Desviación estándar.

El último punto, se refleja sobre todo en los valores de Incremento Promedio Relativo de las variables (Cuadro 4), considerándose que durante los primeros 3 años de crecimiento los niveles de B disponible son suficientes para llenar las necesidades de crecimiento de los árboles, mientras que a mayor edad, cuando el aumento en biomasa aérea depende en mayor proporción de la producción de madera (Segura et al. 2005), la respuesta al B es más elevada. El incremento relativo de las variables altura, diámetro y volumen es mayor conforme la edad de las plantaciones, excepto para la plantación $\mathrm{C}$, la cual se vio afectada por inundaciones en uno de los bloques y compactación provocada por pastoreo de ganado vacuno. Posiblemente, los niveles de B del suelo llenan las necesidades del crecimiento inicial del jaúl, mientras que no lo hacen cuando los árboles alcanzan un mayor crecimiento y se ven favorecidos por la adición de $\mathrm{B}$ al suelo.

Cabe mencionar que el efecto de carga variable de los suelos pudo afectar la absorción de $\mathrm{B}$, en función de la carga variable de cada uno de los suelos estudiados, efecto no medido en el presente trabajo.

\section{Efecto de la adición de $B$ sobre la concentración foliar de nutrimentos}

Dentro del rango de B aplicado al suelo en las plantaciones de jaúl bajo estudio, no se encontró respuesta de la adición del elemento en cuanto a variaciones de su contenido en el suelo y sobre las variables de crecimiento del jaúl (Cuadros 2 y 4). Por esta razón, se decidió analizar el efecto de la adición de $\mathrm{B}$ sobre las concentraciones foliares de otros elementos (datos no incluidos) y 
las de $\mathrm{B}$ y $\mathrm{N}$, elementos que mostraron tendencia a aumentar con la aplicación de B, aunque no estadísticamente probada. No se pudo hacer análisis estadístico para determinar niveles críticos foliares de B porque no se encontró respuesta a las variables de crecimiento, por lo que prevaleció el criterio de tomar muestras compuestas de las 3 repeticiones para cada nivel de B aplicado al suelo y observar así su tendencia. En el Cuadro 5 , puede notarse que para la mayoría de las plantaciones de jaúl bajo estudio, el B foliar tendió a aumentar ligeramente conforme se elevó la cantidad de B adicionada al suelo. En el caso del N foliar, no se encontró ninguna tendencia.

Cuadro 5. Variación de la concentración foliar de B y N en plantaciones de jaúl 18 meses después de aplicar niveles crecientes de B al suelo.

\begin{tabular}{|c|c|c|c|c|c|c|}
\hline \multirow{3}{*}{ Finca } & \multicolumn{6}{|c|}{ B y $\mathrm{N}$ foliar antes y después de aplicar los tratamientos $\left(\mathrm{mg} \cdot \mathrm{kg}^{-1}\right)$} \\
\hline & \multirow{2}{*}{$\frac{\text { Inic. Exp. }}{0}$} & \multicolumn{5}{|c|}{18 meses después de inicio experimento } \\
\hline & & 0 & 2 & 4 & 6 & 8 \\
\hline \multicolumn{7}{|c|}{ B foliar (\%) } \\
\hline $\mathrm{HC}$ & $8 \pm 1$ & 9 & 12 & 14 & 15 & 15 \\
\hline LE & $11 \pm 1$ & 11 & 2 & 8 & 10 & 11 \\
\hline $\mathrm{RMC}$ & $14 \pm 1$ & 8 & 11 & 10 & 13 & 12 \\
\hline $\mathrm{L}$ & $22 \pm 3$ & 13 & 15 & 14 & 16 & 15 \\
\hline $\mathrm{C}$ & $15 \pm 3$ & 14 & 14 & 16 & 17 & 15 \\
\hline \multicolumn{7}{|c|}{$\mathrm{N}$ foliar $(\%)$} \\
\hline $\mathrm{HC}$ & $3,96 \pm 0,19$ & 3,82 & 3,78 & 3,87 & 3,64 & 3,66 \\
\hline LE & $4,10 \pm 0,21$ & 4,01 & 3,93 & 4,1 & 4,27 & 3,95 \\
\hline RMC & $3,65 \pm 0,22$ & 3,56 & 3,70 & 3,88 & 3,82 & 3,60 \\
\hline $\mathrm{L}$ & $3,60 \pm 0,16$ & 4,11 & 4,20 & 4,21 & 4,14 & 3,88 \\
\hline $\mathrm{C}$ & $3,21 \pm 0,17$ & 3,75 & 3,68 & 3,83 & 3,86 & 3,62 \\
\hline
\end{tabular}

Aunque no se comprobó, la poca respuesta foliar a la aplicación de B al suelo puede deberse a que aunque aumentaron los niveles de B aumentaron en el mismo, la absorción del elemento se vio limitada por otras variables ambientales, como la concentración de Ca disponible (efecto antagónico) o una mayor cantidad de arcilla (mayor retención del elemento), casos de las plantaciones Carter y Rosa Marta Cubero (Cuadro 2).

\section{CONCLUSIONES}

El B adicionado al suelo en Andisoles, incrementa su disponibilidad para las plantas, incremento que puede detectarse al extraerlo con agua caliente presurizada o con el método de Mehlich 3. Los valores de correlación entre el B aplicado y el B extraído por el método de Mehlich 3 presentan mejores valores de correlación que los obtenidos con el método de agua caliente presurizada.

A pesar de la respuesta de varias especies forestales y de cultivos comerciales a la adición de $\mathrm{B}$ en Andisoles, la incorporación de 0 a 8 $\mathrm{kg} \cdot \mathrm{ha}^{-1}$ del elemento, no causó ningún incremento en las variables dasométricas del jaúl en 5 sitios de diferente edad, excepto por un incremento significativo en volumen de madera en la plantación de Landelina (8 años de edad). La poca o ninguna respuesta encontradas, se atribuye a que el contenido inicial de B disponible en el suelo osciló entre 0,19-1,00 mg.kg-1, valores en su mayoría sobre el valor crítico de $0,5 \mathrm{mg} \cdot \mathrm{kg}^{-1}$ mencionado para cultivos en Andisoles y a un bajo requerimiento de B por parte del jaúl.

La concentración foliar de B foliar tendió a aumentar ligeramente conforme se elevó la cantidad de B adicionada al suelo, mientras que 
la concentración foliar de $\mathrm{N}$ y otros elementos no mostraron ninguna tendencia.

\section{AGRADECIMIENTOS}

Los autores desean expresar su agradecimiento a Wilbert Rodríguez por su colaboración en los análisis de laboratorio en el CIA, a Fabio Blanco por su ayuda en el análisis estadístico de los datos, a Javier Murillo quien colaboró en medición de las variables dasométricas y el análisis de los resultados. A la CNFL por el financiamiento de esta investigación.

\section{LITERATURA CITADA}

ARAYA J.F. 1978. Efecto del boro en la producción y calidad de coliflor (Brassica oleracea var. botrytis) en San Gerardo de Cot, Cartago. Tesis de Ingeniero Agrónomo, Universidad de Costa Rica. San José, Costa Rica. 48 p.

BERGER K.C., TRUOG E. 1939. Boron determination in soils and plants. Industrial \& Engineering Chemistry Analytical Edition 11:540-545.

BORNEMISZA E. 1982. Introducción a la química de suelos. Secretaría General Organización Estados Americanos, Programa Regional Desarrollo Científico Tecnológico. Serie de Química, Monografía No. 25. Washington, D.C. 74 p.

BRICEÑO J., PACHECO R. 1984. Métodos analíticos para el análisis de suelos y plantas. UCR-CIA. San José, Costa Rica. p 27.

CAMACHO P., MURILLO O. 1986. Algunos resultados preliminares de la epidometría del jaúl Alnus acuminata (H.B.K.) O. Kundze. Departamento de Ingeniería Forestal, Instituto Tecnológico de Costa Rica. Cartago, Costa Rica. p.i.

CANNON P.G. 1981a. Fertilización de plantaciones con NPK, calfos y bórax: resultados al final de cuatro años. Cartón de Colombia, S.A., Cali. 10 p. Informe de Investigación, N ${ }^{\circ}$. 68.

CANNON P.G. 1981b. Respuesta de Pinus kesiya y Pinus oocarpa a la fertilización con varios niveles de calfos, urea, NPK y bórax en la finca Aguaclara al finalizar dos años. Cartón de Colombia, S.A., Cali. 7 p. Informe de Investigación, N ${ }^{\circ} .70$.
CHAVES M.A., ALVARADO A. 1994. Manejo de la fertilización en plantaciones de caña de azúcar (Saccharum spp) en Andisoles de ladera de Costa Rica. In. 15 Congreso Mundial de la Ciencia del Suelo. Acapulco, México. Commission VI, Symposia. Volúmen $7^{\text {a: }}$ 353-372.

DÍAZ-ROMEU R., HUNTER A. 1978. Metodología de muestreo de suelos, análisis químico de suelos y tejido vegetal e investigación en invernadero. CATIE. Turrialba, Costa Rica. pp. 8-27.

FORSYTHE W. 1975. Física de Suelos. Manual de Laboratorio. IICA. San José, Costa Rica. 211 p.

GOLDBERG S., SU C. 2007. New advances in boron soil chemistry. pp. 313-330. In: F. Xu, H.E. Goldbach, P.H. Brown, Bell, R.W. Fujiwara, T. Hunt, C.D. Goldberg, S y Shi L. (eds.). Advances in plant and animal boron nutrition. Proceedings of the $3 \mathrm{ed}$. International symposium on all aspects of plant and animal boron nutrition. Springer.

GONZALES C., KONOW V., LACHICA M. 1983. El Pinus radiata $\mathrm{D}$. Don en Chile: ensayos de fertilización con cobre y boro. Anales de Edafología y Agrobiología 42:1599-1613.

HENRÍQUEZ C., BERTSCH F., SALAS R. 1995. Fertilidad de suelos: Manual de laboratorio. Asociación Costarricense Ciencias del Suelo. San José, Costa Rica. 64 p.

HERNÁNDEZ J.M. 1977. Efecto del boro en la producción y calidad de bróculi (Brassica oleracea var. italica). Tesis de Ingeniero Agrónomo, Universidad de Costa Rica, San José, Costa Rica. 48 p.

JIMÉNEZ E., CORDERO A. 1988. Respuesta de la coliflor (Brassica oleracea var. botrytis) a la fertilización con fósforo, boro y azufre en un suelos Typic Hydrandept en Fraijanes, Costa Rica. In Resúmenes $34^{\circ}$ Reunión PCCMCA. San José, Costa Rica. p. 110.

JUMKOV M. 1994. Evaluación de muestreo del bosque. Guía de campo para extensionistas, UICN/ORMA. San José, Costa Rica. pi.

LADRACH W.E. 1980a. Efecto de la aplicación de diferentes niveles de bórax y N-P-K (10-30-10) en el crecimiento de Cupressus lusitanica al año de su establecimiento. Cartón de Colombia, S.A., Cali. 5 p. Informe de Investigación, $\mathrm{N}^{\circ} .55$.

LADRACH W.E. 1980b. Respuesta al crecimiento de algunos árboles con la aplicación de fósforo, nitrógeno y boro al momento de la plantación en el Cauca y en El Valle. Cartón de Colombia, S.A., Cali. 17 p. Informe de Investigación, $\mathrm{N}^{\circ} .59$. 
LEHTO T., RUUHOLA T., DELL B. 2010. Boron in forest trees and forest ecosystems. Forest Ecology and Management. 260:2053-2069.

MANUAL DIGESTION APPLICATION NOTES. 2001. Milestone, Microwave Laboratory Systems. USA.

MEHLICH A. 1984. Mehlich 3 soil extractant: a modification of Mehlich 2 extractant. Communications in Soil Science and Plant Analysis. 15:1409-1416.

MONTOYA A. 1999. Plan de reforestación para la plantación de Dorval S.A. FUNDECOR. San José, Costa Rica. 233 p.

OPERATION MANUAL. MICROWAVE ACCELERATED REACTION SYSTEM. 2001. MARS 5. CEM Corporation Innovators in Microwave Technology. USA.

PÉREZ V.M. 1957. Algunas deficiencias minerales del cafeto en Costa Rica. Ministerio de Agricultura e Industrias-STICA Informe Técnico N ${ }^{\circ}$. 2. San José, Costa Rica. 27 p.

PÉREZ V.M., CHAVERRI G., BORNEMISZA E. 1957. Algunos aspectos del abonamiento del cafeto con boro y calcio en las condiciones de la Meseta Central de Costa Rica. Ministerio de Agricultura e Industrias-STICA. Informe Técnico No. 1. San José, Costa Rica. 14 p.

RAMÍREZ J.E. 1998. Respuesta del cafeto a la aplicación de dosis de boro al suelo en dos y tres épocas, en Andisoles de Heredia, Costa Rica. Agronomía Costarricense 22(1):19-26.

RÍMOLO L.A. 1970. Efecto del encalado sobre la disponibilidad y adsorción de boro en seis suelos de Costa Rica. Tesis de licenciatura, Universidad de Costa Rica. San José, Costa Rica. 45 p.

SEGURA M., CASTILlO A., ALVARADO A., BLANCO F. 2005. Extracción de nutrimentos en plantaciones de jaúl (Alnus acuminata) en la cuenca del río Virilla, Costa Rica. Agronomía Costarricense 29(2):109-120.

SEGURA M., CASTILlO A., ALVARADO A., BLANCO F. 2006. Variación del contenido foliar de nutrimentos de Alnus acuminata. Agronomía Costarricense 30(1):53-63.

SHOJI S., NANZYO M., DAHLGREN R.A. 1993. Volcanic ash soils, genesis, properties and utilization. ELSEVIER. Developments in Soil Science 21. 288 p.

STONE E.L. 1990. Boron deficiency and excess in forest trees: a review. Forest Ecology and Management 37:49-75.

TOLLENAAR D. 1966. Boron deficiency in cacao, bananas and other crops on volcanic soils of Ecuador. Netherlands Journal Agricultural Science 14(2):138-151. 